TRANSACTIONS OF THE

AMERICAN MATHEMATICAL SOCIETY

Volume 351, Number 8, Pages 3301-3314

$\mathrm{S}$ 0002-9947(99)02222-9

Article electronically published on March 29, 1999

\title{
AN ESTIMATE FOR A FIRST-ORDER RIESZ OPERATOR ON THE AFFINE GROUP
}

\author{
PETER SJÖGREN
}

\begin{abstract}
On the affine group of the line, which is a solvable Lie group of exponential growth, we consider a right-invariant Laplacian $\Delta$. For a certain right-invariant vector field $X$, we prove that the first-order Riesz operator $X \Delta^{-1 / 2}$ is of weak type $(1,1)$ with respect to the left Haar measure of the group. This operator is therefore also bounded on $L^{p}, 1<p \leq 2$. Locally, the operator is a standard singular integral. The main part of the proof therefore concerns the behaviour of the kernel of the operator at infinity and involves cancellation.
\end{abstract}

\section{INTRODUCTION AND RESULTS}

Let $G$ be the Lie group of positively oriented affine maps $\mathbb{R} \rightarrow \mathbb{R}$. Such a map can be written $\xi \rightarrow e^{t} \xi+s$. Thus $G$ consists of the points $x=(s, t) \in \mathbb{R}^{2}$, with group multiplication $(s, t)\left(s^{\prime}, t^{\prime}\right)=\left(s+e^{t} s^{\prime}, t+t^{\prime}\right)$. This solvable Lie group is not unimodular; indeed $d m(x)=e^{-t} d s d t$ is the left Haar measure, whereas the right Haar measure $d m_{r}(x)=d s d t$ is Lebesgue measure. The modular function is thus $\delta(s, t)=e^{-t}$. In this paper we shall use $m$. Notice that the lack of unimodularity implies that $G$ is of exponential growth.

The Lie algebra $\mathfrak{g}$ of $G$ is spanned by the right-invariant fields

$$
X=\partial / \partial s \text { and } Y=\partial / \partial t+s \partial / \partial s .
$$

The operator $-X^{2}-Y^{2}$ on $C_{0}^{\infty}(G)$ has a self-adjoint closure $\Delta$ on $L^{2}=L^{2}(G, m)$. As seen in Gaudry, Qian and Sjögren [8], $\Delta$ is positive definite and one-to-one. Thus its powers $\Delta^{\alpha}, \alpha \in \mathbb{R}$, have dense domains and are self-adjoint. This makes it possible to form Riesz operators like $X \Delta^{-1 / 2}$, which is the operator studied in this paper.

Before stating our results, we sketch some background and context. Many authors have studied Riesz operators and their $L^{p}$ boundedness properties on various Lie groups, symmetric spaces and Riemannian manifolds. For nilpotent Lie groups and first-order Riesz operators, the boundedness on $L^{p}, 1<p<\infty$, and the weak type $(1,1)$ was proved by Lohoué and Varopoulos [15]. This was extended to all groups of polynomial growth by Saloff-Coste [17] and Alexopoulos [1]. In [1] it is also proved that a second-order Riesz operator of the form $Z_{1} Z_{2} \Delta^{-1}$ or $\Delta^{-1} Z_{1} Z_{2}$ with $Z_{1}, Z_{2} \in \mathfrak{g} \backslash\{0\}$ is not bounded on any $L^{p}$ space.

Received by the editors December 15, 1996 and, in revised form, August 15, 1997.

1991 Mathematics Subject Classification. Primary 43A80, 42B20; Secondary 22E30.

(C)1999 American Mathematical Society 
Work by Lohoué [14] and Burns, ter Elst and Robinson [5] gives analogous results for all nonamenable groups. The latter paper also contains quite general results when $\Delta$ is replaced by $\Delta+c I, c>0$.

In the setting of symmetric spaces of noncompact type, Anker [2] considered Riesz operators associated with the Laplace-Beltrami operator. He obtained the weak type $(1,1)$ estimate for first-order operators and the $L^{p}$ estimates for operators of arbitrary order. Lohoué [13] and Bakry [3] dealt with Riesz operators on certain Riemannian manifolds. The setting of Coulhon and Duong [6] is a Riemannian manifold for which the balls satisfy the doubling condition with respect to volume. It is interesting that they prove the $L^{p}$ boundedness for $1<p \leq 2$ but disprove it for $2<p<\infty$.

The papers mentioned above do not apply to our setting, the affine group $G$. Second-order Riesz operators on $G$ were studied in Gaudry, Qian and Sjögren [8]. It was proved that for any $Z_{1}, Z_{2} \in \mathfrak{g}$ the operator $Z_{1} \Delta^{-1} Z_{2}$ is bounded on $L^{p}, 1<p<$ $\infty$, and of weak type $(1,1)$. Further, $Z_{1} Z_{2} \Delta^{-1}$ and $\Delta^{-1} Z_{1} Z_{2}$ with $Z_{1}, Z_{2} \neq 0$ were seen to have none of these boundedness properties. These results were extended to any solvable $N A$ group coming from the Iwasawa decomposition of a rank 1 semisimple group in Gaudry and Sjögren [9]. In the complex affine group, treated in Gaudry and Sjögren [10], the second-order Riesz operators turn out to have slightly different boundedness properties.

As for first-order Riesz operators, it is a general, simple fact that $Z \Delta^{-1 / 2}$ and $\Delta^{-1 / 2} Z, Z \in \mathfrak{g}$, are bounded on $L^{2}$. A proof can be found for instance in $[8$, Lemma 4].

The aim of this paper is to prove that the operator $X \Delta^{-1 / 2}$ is of weak type $(1,1)$, and hence bounded on $L^{p}$ for $1<p \leq 2$. Except for the $L^{2}$ case, this seems to be the first result obtained for a first-order Riesz operator in a group of exponential growth. The operator $X \Delta^{-1 / 2}$ is in fact also bounded on $L^{p}$ for $2<p<\infty$; this is proved in a recent paper [11] by Gaudry and Sjögren. The main result of [11] is actually the weak type $(1,1)$ of the adjoint $\Delta^{-1 / 2} X$. We point out that the corresponding questions with the field $X$ replaced by $Y$ are still open.

The kernels of the Riesz operators usually behave at the origin like a standard singular integral kernel, and then the local parts of the operators always have the boundedness properties studied. The main issue is the global part of the operator. In [8] and [9] the kernel of $Z_{1} \Delta^{-1} Z_{2}$ was found to be integrable at infinity, which immediately implies the boundedness properties of the global part. This does, however, not hold for $X \Delta^{-1 / 2}$. Our proof of the weak type $(1,1)$ relies on cancellation between positive and negative parts at infinity. We therefore start by computing the kernel rather explicitly. This computation is done via a known expression for the resolvent. Another possibility is to use the heat kernel as in [9]. Notice that this requires very precise knowledge about the asymptotic behaviour of the heat kernel.

We need some notation to state the theorems. The group $G$ has a Riemannian symmetric space structure, that of the hyperbolic disc or half-space. The Riemannian distance is invariant under left translation and given by

$$
\cosh d(x, e)=z, \text { where } z=z(x)=\frac{1}{2}\left(e^{t}+e^{-t}+s^{2} e^{-t}\right) .
$$

Given a kernel in $G$, we define its principal value by deleting as usual a small ball $\{x:|x|<\rho\}$ centred at $e=(0,0)$ and letting $\rho \rightarrow 0$. Here we let $|x|=\left(s^{2}+t^{2}\right)^{1 / 2}$, 
although one could also use the distance $d$. By $\mathfrak{Q}_{\nu}^{\mu}$ we denote the Legendre function of the second kind.

The following is our main result.

Theorem 1. Let

$$
K(x)=\frac{2}{\pi} s e^{-t / 2} \int_{0}^{\infty}\left(1-z^{2}\right)^{-1 / 2} \mathfrak{Q}_{\lambda-1 / 2}^{1}(z) d \lambda .
$$

The operator defined by convolution from the left with the distribution $\mathrm{pv} K$ is bounded on $L^{p}(m)$ for $1<p \leq 2$ and of weak type $(1,1)$ with respect to $m$. For $f \in D\left(\Delta^{-1 / 2}\right)$ one has $X \Delta^{-1 / 2} f=\operatorname{pv} K * f$, where the derivative $X$ is taken in the sense of distributions. Thus $X \Delta^{-1 / 2}$ extends to an operator which is bounded on $L^{p}, 1<p \leq 2$, and of weak type $(1,1)$.

The kernel $K$ behaves locally like a singular integral kernel of Calderón-Zygmund type; cf. Lemma 5 . The essential difficulty in proving Theorem 1 occurs at infinity. To prove the weak type inequality for pv $K$, we therefore first compute, in Section 2 , the asymptotic behaviour of $K$ at infinity. Except for integrable terms, $K$ is at infinity a multiple of the kernel $S$ defined in the following theorem, with $r(s)=$ $s /\left(1+s^{2}\right)^{3 / 2}$.

Theorem 2. Let $r \in C^{1}(\mathbb{R})$ satisfy $r(s)=O\left(|s|^{-1-\epsilon}\right)$ and $r^{\prime}(s)=O\left(|s|^{-2-\epsilon}\right)$ as $s \rightarrow \pm \infty$ for some $\epsilon>0$, and assume that $\int r(s) d s=0$. Then left convolution with the kernel

$$
S(s, t)=r(s) e^{t} t^{-1} \chi_{\{t<-1\}}
$$

defines an operator of weak type $(1,1)$ for $m$.

As can be seen from Section 3, Theorem 2 is the main ingredient in the proof of Theorem 1. The kernel $S$ is rather similar to those treated by Strömberg [20] and [21]. He proves that convolution from the right with the kernel $S_{1}(s, t)=$ $r(s) e^{t} \chi_{\{t<-1\}}$ defines an operator of weak type $(1,1)$, for an arbitrary integrable function $r$. This is implicit in [21] and explicit in [20, Theorem 1]. But to obtain a left convolution kernel of weak type $(1,1)$, we need both the small factor $t^{-1}$ in $S$ and the cancellation coming from the vanishing moment of $r$. Indeed, it follows from [8, Lemmas 11, 13 and 14] that our Theorem 2 would be false without the factor $t^{-1}$. An example at the end of Section 4 shows that $r(s)$ cannot be replaced by $|r(s)|$ in Theorem 2. It is worth observing that $S_{1}$ is in weak $L^{1}$ and that $S$ is in the smaller space weak $L \log L$, but neither kernel is integrable.

To prove Theorem 2, we shall reduce the convolution in $G$ to a convolution in $\mathbb{R}$. A similar reduction argument was used in $[8$, Lemma 11] to disprove the weak type $(1,1)$ estimate when $t^{-1}$ is omitted in $S$.

The next step in [8] after this reduction consists in disproving the implication

$$
f \in L^{1}(\mathbb{R}) \Rightarrow r_{y} * f(x) \in L^{1, \infty}(\mu),
$$

where $\mu$ is the measure $y^{-1} d x d y$ in $\{(x, y): x \in \mathbb{R}, y>0\}$. Here $r$ is as in our Theorem 2, and $r_{y}(x)=y^{-1} r(x / y)$. The proof of Theorem 2 by contrast goes via a weaker, true version of (2), which we state as Theorem 3 and in which $y$ is restricted to a compact subinterval of $\mathbb{R}_{+}$. Section 4 contains the argument needed to reduce Theorem 2 to Theorem 3 . 
Theorem 3. Let $r$ be as in Theorem 2, and take $N \in \mathbb{N}$ and $a>0$. For each $f \in L^{1}(\mathbb{R})$, the function $(x, y) \rightarrow r_{y} * f(x)$ is in weak $L^{1}$ with respect to $\mu$ in the set $\mathbb{R} \times\left[a, e^{N} a\right]$, with quasinorm bounded by $C \sqrt{N}\|f\|_{1}$. Here $C$ depends only on $r$.

In this result, the factor $\sqrt{N}$ is best possible, as shown by the counterexample to (2) given in [8, Lemma 14]. The author's first proof of Theorem 3 went via a discretised version of it, equivalent to the following inequality for (dyadic) martingales. Let $P$ denote a probability measure and $\left(X_{n}\right)$ the conditional expectations of the integrable random variable $X$, with respect to an increasing sequence of $\sigma$-algebras. Then

$$
\sum_{1}^{N} P\left(\left|X_{n}-X_{n-1}\right|>\lambda\right) \leq \frac{C \sqrt{N}}{\lambda}\|X\|_{1}, \quad \lambda>0 .
$$

The author's proof of the discretised version of Theorem 3 was mainly analytical. S. Janson then gave a short proof of (3) based on the martingale square function. This inspired the author to an analogous, short proof of Theorem 3 by means of a $g$-function and without discretisation. This argument is presented in Section 5.

The author is grateful to Garth Gaudry and Tao Qian for stimulating discussions and valuable advice.

\section{Preliminaries}

In this section we explain some notation, give a simple lemma about integrability with respect to $m$, and estimate the kernel $K$.

By $\|\cdot\|_{p}$ we denote the norm both in $L^{p}(\mathbb{R})$ and in $L^{p}(G, m)$. The quasinorm of weak $L^{1}$ is written $\|\cdot\|_{1, \infty}$. For functions defined in $G$, the measure used will be $m$ unless otherwise explicitly stated. In particular, $\langle\cdot, \cdot\rangle$ is the scalar product in $L^{2}(G, m)$. With $f$ defined in $G$, we write $\check{f}(x)=f\left(x^{-1}\right)$, so that in particular $\|\check{f}\|_{L^{1}\left(d m_{r}\right)}=\|f\|_{1}$. By $c>0$ and $C<\infty$ we denote many different constants, and $f \sim g$ means $c \leq f / g \leq C$. Notice that for $x \in G$ close to $e$, we have

$$
d(x, e) \sim \sqrt{z(x)-1} \sim|x|=\left(s^{2}+t^{2}\right)^{1 / 2} .
$$

For $\lambda \in \mathbb{C} \backslash(-\infty, 0]$, the resolvent $(\lambda+\Delta)^{-1}$ is bounded on $L^{2}$ and given by convolution from the left with an integrable kernel $k_{\lambda}$. This kernel is

$$
k_{\lambda}(x)=e^{t / 2} \mathfrak{Q}_{\sqrt{\lambda}-1 / 2}^{0}(z)
$$

with $\Re \sqrt{\lambda}>0$, as proved by Hulanicki [12].

Lemma 4. Let $j \geq 0, a \in \mathbb{R}$ and $b \geq 0$. Then the function $G(s, t)=s^{j} e^{a t} z^{-b}$ belongs to $L^{1}(x \in G: z>2)$, provided that $a+b>1$ and $j+a<b$. Similarly, $G(s, t)(\log z)^{-\gamma}$ and $G(s, t)(\log (2+|t|))^{-\gamma}$ belong to the same space for $\gamma>1$, when $a+b \geq 1$ and $j+a<b$ and also when $a+b>1$ and $j+a \leq b$.

Proof. This is straightforward. For $t>0$ and $|s|<e^{t}$ one has $z \sim e^{t}$, and otherwise $z \sim\left(1+s^{2}\right) e^{-t}$. One observes that the inequality $2 b>j+1$ is a consequence of the hypotheses, even in the cases with a logarithmic factor. The rest can be left to the reader.

Next we consider some kernels on $G$. As we shall see in Section 3,

$$
U(x)=\frac{2}{\pi} e^{t / 2} \int_{0}^{\infty} \mathfrak{Q}_{\lambda-1 / 2}^{0}(z) d \lambda
$$


is the kernel of the operator $\Delta^{-1 / 2}$. Differentiating under the integral sign and using the formula for derivatives of Legendre functions, see Magnus and Oberhettinger [16, IV.5c], we obtain $K=\partial U / \partial s$. We shall derive some estimates for $U$ and its derivatives near $e$ and near $\infty$.

From [16, IV.5e, first formula on page 68] we have for $\lambda>0$ and $z>1$

$$
\mathfrak{Q}_{\lambda-1 / 2}^{0}(z)=2^{-\lambda-1 / 2} \int_{-1}^{1}\left(1-u^{2}\right)^{\lambda-1 / 2}(z-u)^{-\lambda-1 / 2} d u
$$

Write $M=2(z-u) /\left(1-u^{2}\right)$ and observe that $M>2 /(1+u)>1$ for $z>1,|u|<1$. Thus

$$
\begin{aligned}
U(x) & =\frac{\sqrt{2}}{\pi} e^{t / 2} \int_{-1}^{1}\left(1-u^{2}\right)^{-1 / 2}(z-u)^{-1 / 2} d u \int_{0}^{\infty} M^{-\lambda} d \lambda \\
& =\frac{\sqrt{2}}{\pi} e^{t / 2} \int_{-1}^{1}\left(1-u^{2}\right)^{-1 / 2}(z-u)^{-1 / 2}(\log M)^{-1} d u
\end{aligned}
$$

Now insert the expression for $z$ from (1). One verifies that it is possible to differentiate under the integral sign, so that

$$
\begin{aligned}
K & =\frac{\partial U}{\partial s} \\
& =-\frac{\sqrt{2}}{\pi} s e^{-t / 2} \int_{-1}^{1}\left(1-u^{2}\right)^{-1 / 2}(z-u)^{-3 / 2}\left(\frac{1}{2}(\log M)^{-1}+(\log M)^{-2}\right) d u .
\end{aligned}
$$

For the second derivatives, we observe that for small $s, t$ we have the estimates

$$
\begin{aligned}
\left|\frac{\partial^{2} U}{\partial s^{2}}(x)\right| & +\left|\frac{\partial^{2} U}{\partial s \partial t}(x)\right| \\
& \leq C \int_{-1}^{1}\left(1-u^{2}\right)^{-1 / 2}(z-u)^{-3 / 2}\left((\log M)^{-1}+(\log M)^{-2}\right) d u \\
& +C\left(s^{2}+t^{2}\right) \int_{-1}^{1}\left(1-u^{2}\right)^{-1 / 2}(z-u)^{-5 / 2}\left((\log M)^{-1}+(\log M)^{-3}\right) d u .
\end{aligned}
$$

Lemma 5. As $x \rightarrow e, x \in G$, one has

$$
\begin{gathered}
U(x)=O\left(|x|^{-1}\right), \\
\left|\frac{\partial U}{\partial s}(x)\right|=O\left(|x|^{-2}\right)
\end{gathered}
$$

and

$$
\left|\frac{\partial^{2} U}{\partial s^{2}}(x)\right|+\left|\frac{\partial^{2} U}{\partial s \partial t}(x)\right|=O\left(|x|^{-3}\right) .
$$

Proof. To obtain these three estimates, it will be enough to prove that for $j=1,2,3$ and $1 \leq j^{\prime} \leq j$

$$
\int_{-1}^{1}\left(1-u^{2}\right)^{-1 / 2}(z-u)^{1 / 2-j}(\log M)^{-j^{\prime}} d u=O\left((z-1)^{1 / 2-j}\right)
$$

as $z \searrow 1$. We split $[-1,1]$ into subintervals and estimate the corresponding parts of this integral. For $-1<u<0$ both $z-u$ and $\log M$ are bounded away from 0 , 
so that

$$
\int_{-1}^{0} \leq C
$$

Let $\xi=z-1$. For $1-\xi<u<1$ one has $M>4(1-u) /\left(1-u^{2}\right)>2$, and so

$$
\int_{1-\xi}^{1} \leq C \xi^{1 / 2-j} \int_{1-\xi}^{1}\left(1-u^{2}\right)^{-1 / 2} d u \leq C \xi^{1-j} .
$$

In the remaining interval $0<u<1-\xi$, we write

$$
M=1+\frac{2 \xi}{1-u^{2}}+\frac{1-u}{1+u} .
$$

It follows that $M$ stays bounded for these $u$ and $\xi$, and so it is enough to take $j^{\prime}=j$ in (6). Clearly $\log M \geq c \max (\xi /(1-u), 1-u)$, which implies

$$
\int_{0}^{1-\sqrt{\xi}} \leq \int_{0}^{1-\sqrt{\xi}}(1-u)^{-\frac{1}{2}+\frac{1}{2}-j-j} d u=O\left(\xi^{\frac{1}{2}-j}\right)
$$

and

$$
\int_{1-\sqrt{\xi}}^{1-\xi} \leq \xi^{-j} \int_{1-\sqrt{\xi}}^{1-\xi}(1-u)^{-\frac{1}{2}+\frac{1}{2}-j+j} d u=O\left(\xi^{\frac{1}{2}-j}\right) .
$$

This proves (6) and the lemma.

We next estimate $U$ and $K$ at infinity.

Lemma 6. Let $r(s)=s /\left(1+s^{2}\right)^{3 / 2}$ in the expression for $S$ given in Theorem 2. Then $K-2 S \in L^{1}\{x \in G: z>2\}$.

Proof. We shall prove this by subtracting integrable terms from $K$ until the remainder is $2 S$. In the expression (5) for $K$, the term $(\log M)^{-2}$ gives rise to a part of $K$ which is $O\left(s e^{-t / 2} z^{-3 / 2}(\log z)^{-2}\right)$ as $x \rightarrow \infty$ and thus integrable in view of Lemma 4.

For the term $\frac{1}{2}(\log M)^{-1}$, we use the simple fact that $1 /(x+y)=1 / x+O\left(y / x^{2}\right)$ for $x>0$ and $-x / 2<y<\infty$, getting

$$
\begin{aligned}
(\log M)^{-1} & =\frac{1}{\log (z-u)+\log \left(2 /\left(1-u^{2}\right)\right)}=\frac{1}{\log z+O\left(z^{-1}\right)+\log \left(2 /\left(1-u^{2}\right)\right)} \\
& =\frac{1}{\log z}+O\left(\frac{z^{-1}+\log \left(2 /\left(1-u^{2}\right)\right)}{(\log z)^{2}}\right), \quad z \rightarrow \infty
\end{aligned}
$$

uniformly in $|u|<1$. To deal with the last term here, notice that

$$
\begin{aligned}
& |s| e^{-t / 2} \int_{-1}^{1}\left(1-u^{2}\right)^{-1 / 2}(z-u)^{-3 / 2} \frac{z^{-1}+\log \left(2 /\left(1-u^{2}\right)\right)}{(\log z)^{2}} d u \\
\leq & C|s| e^{-t / 2} z^{-5 / 2}+C|s| e^{-t / 2} z^{-3 / 2}(\log z)^{-2},
\end{aligned}
$$

which is in $L^{1}\{z>2\}$. The expression in (5) is thus

$$
-\frac{1}{\pi \sqrt{2}} s e^{-t / 2} \int_{-1}^{1}\left(1-u^{2}\right)^{-1 / 2}(z-u)^{-3 / 2}(\log z)^{-1} d u+\text { integrable terms }
$$


in $\{z>2\}$. Here we can replace $(z-u)^{-3 / 2}$ by $z^{-3 / 2}$, with an integrable error. The result is that $K$ differs from

$$
-\frac{1}{\pi \sqrt{2}} s e^{-t / 2} z^{-3 / 2}(\log z)^{-1} \int_{-1}^{1}\left(1-u^{2}\right)^{-1 / 2} d u=-\frac{1}{\sqrt{2}} s e^{-t / 2} z^{-3 / 2}(\log z)^{-1}
$$

by a function in $L^{1}\{z>2\}$.

Arguing as in Lemma 4 , we see that $s e^{-t / 2} z^{-3 / 2}(\log z)^{-1}$ is integrable in $\{x \in$ $G: z>2, t>-1\}$. Thus it is enough to consider the kernel

$$
K_{1}=-\frac{1}{\sqrt{2}} s e^{-t / 2} z^{-3 / 2}(\log z)^{-1} \chi_{\{t<-1\}} .
$$

If we replace $z^{-3 / 2}$ in $K_{1}$ by $2^{3 / 2}\left(1+s^{2}\right)^{-3 / 2} e^{3 t / 2}$, we see from (1) that the error is at most $C|s|\left(1+s^{2}\right)^{-5 / 2} e^{3 t} \chi_{\{t<-1\}}$, hence integrable.

Since $\log z=-t+\log \left(1+s^{2}\right)-\log 2+O\left(e^{2 t}\right)$ as $t \rightarrow-\infty$, we get $\log z>-t / C$ for $t<-1$, and so

$$
\left|\frac{1}{\log z}+\frac{1}{t}\right| \leq C \frac{\log \left(2+s^{2}\right)}{t^{2}} .
$$

Thus if we finally replace $1 / \log z$ by $-1 / t$ in $K_{1}$, the error is at most

$$
C|s| e^{-t / 2}\left(1+s^{2}\right)^{-3 / 2} e^{3 t / 2} t^{-2} \log \left(2+s^{2}\right) \chi_{\{t<-1\}} .
$$

This expression is in $L^{1}$. Since the kernel we have now arrived at equals $2 S$, this ends the proof of Lemma 6 .

\section{Proof that Theorem 2 implies Theorem 1}

We start by deriving the kernels of $\Delta^{-1 / 2}$ and $X \Delta^{-1 / 2}$. Let $V=\{\Delta u: u \in$ $\left.C_{0}^{\infty}\right\} \subset C_{0}^{\infty}$. In [9, Theorem 5] it is verified that $V$ is a dense subspace of $L^{2}$. Notice that $V \subset D\left(\Delta^{-1}\right) \subset D\left(\Delta^{-1 / 2}\right)$.

The operator $\Delta$ has a spectral resolution $\left(E_{t}\right)_{0}^{\infty}$. We get for $f \in V$ and $g \in C_{0}^{\infty}$

$$
\left\langle\Delta^{-1 / 2} f, g\right\rangle=\int_{0}^{\infty} t^{-1 / 2} d\left\langle E_{t} f, g\right\rangle .
$$

This integral is absolutely convergent, since

$$
\left(\int_{0}^{\infty} t^{-1 / 2}\left|d\left\langle E_{t} f, g\right\rangle\right|\right)^{2} \leq \int_{0}^{\infty} t^{-1} d\left\langle E_{t} f, f\right\rangle \int_{0}^{\infty} d\left\langle E_{t} g, g\right\rangle<\infty
$$

see Birman and Solomjak [4, 1.5.20 and 5.1.2]. We can therefore insert $t^{-1 / 2}=$ $(2 / \pi) \int_{0}^{\infty} d \lambda /\left(\lambda^{2}+t\right)$ in (7) and apply Fubini's theorem, which yields

$$
\left\langle\Delta^{-1 / 2} f, g\right\rangle=\frac{2}{\pi} \int_{0}^{\infty} d \lambda \int_{0}^{\infty} \frac{d\left\langle E_{t} f, g\right\rangle}{\lambda^{2}+t}=\frac{2}{\pi} \int_{0}^{\infty}\left\langle k_{\lambda^{2}} * f, g\right\rangle d \lambda .
$$

Since $k_{\lambda^{2}} \geq 0$ and $(2 / \pi) \int k_{\lambda^{2}} d \lambda=U$ is locally integrable, we can change the order of integration in the last expression, getting

$$
\left\langle\Delta^{-1 / 2} f, g\right\rangle=\langle U * f, g\rangle .
$$

Thus $U$ is the kernel of $\Delta^{-1 / 2}$ in the sense that $\Delta^{-1 / 2} f=U * f$ for $f \in V$.

When $f \in V$, the convolution $U * f$ is $C^{\infty}$, and

$$
X(U * f)(y)=\int U(x) X_{y} f\left(x^{-1} y\right) d m(x) .
$$


But

$$
X_{y} f\left(x^{-1} y\right)=\left.\frac{d}{d \tau} f\left(x^{-1} \exp (\tau X) y\right)\right|_{\tau=0}=-X_{x} f\left(x^{-1} y\right)
$$

so that

$$
X(U * f)(y)=-\lim _{\rho \rightarrow 0} \int_{|x|>\rho} U(x) X_{x} f\left(x^{-1} y\right) d m(x) .
$$

In this last integral, we integrate by parts in $s$, where $x=(s, t)$ as before. Write $x_{ \pm}(t)=\left( \pm \sqrt{\rho^{2}-t^{2}}, t\right) \in G$ for $|t|<\rho$. The result is

$$
\begin{aligned}
X(U * f)(y) & \\
=\lim _{\rho \rightarrow 0}\left[\int _ { - \rho } ^ { \rho } \left(U\left(x_{+}(t)\right) f\left(x_{+}(t)^{-1} y\right)-\right.\right. & \left.U\left(x_{-}(t)\right) f\left(x_{-}(t)^{-1} y\right)\right) e^{-t} d t \\
& \left.+\int_{|x|>\rho} X U(x) f\left(x^{-1} y\right) d m(x)\right] .
\end{aligned}
$$

The estimate for $U$ in Lemma 5 implies that the first integral here tends to 0 as $\rho \rightarrow 0$, because $U(x)$ is even in $s$ and $f\left(x_{ \pm}(t)^{-1} y\right)=f(y)+O(\rho)$. The last integral tends to pv $X U * f(x)$. Since $X U=K$, we have proved that $X \Delta^{-1 / 2} f=$ pv $K * f$ for $f \in V$.

Let $\phi \in C_{0}^{\infty}(G)$ with $\phi=1$ in a neighbourhood of $e$. Writing pv $K=\operatorname{pv}(\phi K)+$ $(1-\phi) K$, we split pv $K$ into a local part and a global part. Lemma 6 means that the global part differs from $2 S$ by an integrable function. To see that Theorem 1 follows from Theorem 2, we need thus only prove that the local part defines an operator of weak type $(1,1)$. This follows from the next lemma, by standard Calderón-Zygmund arguments, in view of the estimates for the derivatives of $K$ in Lemma 5.

Lemma 7. Left convolution by the kernel $\mathrm{pv}(\phi K)$ is a bounded operator on $L^{2}$, provided that $\phi$ is of the form $\phi=\phi_{1} * \phi_{2}$ with $\phi_{1}, \phi_{2} \in C_{0}^{\infty}$.

It is clear that one can choose $\phi$ of this form. We remark that the lemma actually holds without the assumption $\phi=\phi_{1} * \phi_{2}$. It is introduced because it allows us to give a short proof. This proof is based on a representation formula for the product of two convolutions used in Edwards and Gaudry [7, Proof of Theorem B.2.1, Appendix B, pp. 188-189]. The argument extends to $L^{p}$.

Proof of Lemma \%. Take any two test functions $f, g \in C_{0}^{\infty}$ and write

$$
\left\langle\left(\left(\phi_{1} * \phi_{2}\right) \operatorname{pv} K\right) * f, g\right\rangle=\left\langle\left(\phi_{1} * \phi_{2}\right) \operatorname{pv} K, g * \check{f}\right\rangle=\left\langle\operatorname{pv} K,\left(\phi_{1} * \phi_{2}\right)(g * \check{f})\right\rangle .
$$

Letting subscripts denote right translation, we have

$$
\begin{aligned}
\left(\phi_{1} * \phi_{2}\right)(g * \check{f})(x) & =\int \phi_{1}(y) \phi_{2}\left(y^{-1} x\right) d m(y) \int g(w) \check{f}\left(w^{-1} x\right) d m(w) \\
& =\int \phi_{1}(y) \phi_{2}\left(y^{-1} x\right) \int g(y w) \check{f}\left(w^{-1} y^{-1} x\right) d m(w) d m(y) \\
& =\int d m(w) \int \phi_{1}(y) g_{w}(y) \phi_{2}\left(y^{-1} x\right)\left(f_{w}\right)^{\vee}\left(y^{-1} x\right) d m(y) \\
& =\int d m(w)\left(\phi_{1} g_{w}\right) *\left(\check{\phi}_{2} f_{w}\right)^{\vee}(x) .
\end{aligned}
$$


As pointed out in the introduction, left convolution by $\operatorname{pv} K$ is bounded on $L^{2}$, with norm $A$, say. Therefore,

$$
\begin{aligned}
& \left\langle\operatorname{pv} K,\left(\phi_{1} * \phi_{2}\right)(g * \check{f})\right\rangle \mid \\
\leq & \left|\int d m(w)\left\langle\operatorname{pv} K,\left(\phi_{1} g_{w}\right) *\left(\check{\phi}_{2} f_{w}\right)^{\vee}\right\rangle\right| \\
\leq & \int d m(w)\left|\left\langle\operatorname{pv} K *\left(\check{\phi}_{2} f_{w}\right), \phi_{1} g_{w}\right\rangle\right| \\
\leq & A \int d m(w)\left\|\check{\phi}_{2} f_{w}\right\|_{2}\left\|\phi_{1} g_{w}\right\|_{2} \\
\leq & A\left(\int\left\|\check{\phi}_{2} f_{w}\right\|_{2}^{2} d m(w)\right)^{1 / 2} \\
& \times\left(\int\left\|\phi_{1} g_{w}\right\|_{2}^{2} d m(w)\right)^{1 / 2} \\
\leq & A\left(\iint\left|\check{\phi}_{2}(y) f(y w)\right|^{2} d m(y) d m(w)\right)^{1 / 2} \\
& \times\left(\iint\left|\phi_{1}(y) g(y w)\right|^{2} d m(y) d m(w)\right)^{1 / 2} \\
\leq & A\left\|\check{\phi}_{2}\right\|_{2}\|f\|_{2}\left\|\phi_{1}\right\|_{2}\|g\|_{2} .
\end{aligned}
$$

This concludes the proof of the lemma.

As remarked above, this proves Theorem 1 modulo Theorem 2.

\section{Theorem 3 implies Theorem 2}

We must estimate $S * f$ for $f \in L^{1}(m)$. One finds

$$
S * f(s, t)=\iint_{t+v<-1} r\left(s+e^{t} u\right) e^{t+v} \frac{1}{t+v} \check{f}(u, v) e^{-v} d u d v .
$$

By simply deleting the factor $1 /(t+v)$ and taking absolute values, we first derive a crude estimate. Write for $u \in \mathbb{R}$

$$
F(u)=\int|\check{f}(-u, v)| d v
$$

and $r_{y}(x)=y^{-1} r(x / y)$ for $x \in \mathbb{R}, y>0$. Then

$$
|S * f(s, t)| \leq \int e^{t}\left|r\left(e^{t}\left(e^{-t} s+u\right)\right)\right| F(-u) d u=\left|r_{e^{-t}}\right| * F\left(e^{-t} s\right),
$$

where the second convolution is taken in $\mathbb{R}$. This is essentially Lemma 11 of [8]. Observe that $\|F\|_{1} \leq\|f\|_{1}$. Thus

$$
\int|S * f(s, t)| d s \leq e^{t}\|r\|_{1}\|f\|_{1},
$$

and Chebyshev's inequality implies that for each $t>0$

$$
|\{s:|S * f(s, t)|>\lambda\}| \leq C \lambda^{-1} e^{t}\|f\|_{1} .
$$

Clearly this is not good enough to allow integration with respect to $e^{-t} d t$. Our idea is still to rewrite (9) by means of a one-dimensional convolution in the $s$ variable, but keeping the factor $1 /(t+v)$ and without taking absolute values. There are two 
obstructions. First, the factor $1 /(t+v)$ does not fit into this scheme. We solve this by chopping up the kernel $S$ into parts corresponding to intervals in $t$. In each interval, the factor $1 / t$ in the kernel can be replaced by a constant. The second obstruction is that in (9), or rather in the corresponding formulae with the parts of $S$ just mentioned instead of $S$, the integration in $v$ is not over all of $\mathbb{R}$ but over an interval which depends on $t$. To deal with this, we chop up $f$ by means of intervals in the second variable. These intervals will be much smaller than those used to chop up $S$. When we now convolve a part of $S$ with a part of $f$, the interval of integration in $v$ can in most cases be taken as either all of $\mathbb{R}$, which will produce a convolution in $\mathbb{R}$, or as the empty set, which will produce nothing. The remaining cases will be seen to be so rare that we can afford using (10).

Let us now carry out this program. Define intervals $I_{j}=\left(-(j+1)^{4},-j^{4}\right)$ for $j=1,2, \ldots$. The lengths $\left|I_{j}\right|$ increase like $j^{3}$ for large $j$. Let $\chi_{j}$ be the characteristic function of $I_{j}$. For $t \in I_{j}$ one has $\left|t^{-1}+j^{-4}\right| \leq C j^{3} /\left(|t| j^{4}\right)=O\left(|t|^{-5 / 4}\right)$ as $j \rightarrow \infty$. This means that if we replace $S$ by $\sum_{1}^{\infty} S_{j}$ with $S_{j}=-j^{-4} r(s) e^{t} \chi_{j}(t)$, the error will be controlled by $|r(s)| e^{t}|t|^{-5 / 4} \chi_{\{t<-1\}}$, which is integrable.

Take an $f \in L^{1}(G)$. Fixing $j$, we shall estimate $S_{j} * f$. Divide the line into intervals $J_{i}=\left(i j^{2},(i+1) j^{2}\right), i \in \mathbb{Z}$, and set $f_{i}(s, t)=f(s, t) \chi_{J_{i}}(-t)$. Fixing also $i$, we have

$$
S_{j} * f_{i}(s, t)=-j^{-4} \iint_{t+v \in I_{j}, v \in J_{i}} r\left(s+e^{t} u\right) e^{t+v} \check{f}(u, v) e^{-v} d u d v .
$$

In this integral, $v$ runs over the interval $J_{i} \cap\left(I_{j}-t\right)$. For each $t$, exactly one of the following three cases occurs:

1. $J_{i} \cap\left(I_{j}-t\right)=J_{i}$,

2. $J_{i} \cap\left(I_{j}-t\right)=\emptyset$,

3. $J_{i} \cap\left(I_{j}-t\right)$ is a nonempty proper subset of $J_{i}$.

Let $\chi^{(1)}$ be the characteristic function of the set of $t \in \mathbb{R}$ for which Case 1 occurs, and write $\chi^{(1)}(s, t)=\chi^{(1)}(t)$. Define $\chi^{(3)}$ similarly. Then

$$
S_{j} * f_{i}=\left(S_{j} * f_{i}\right) \chi^{(1)}+\left(S_{j} * f_{i}\right) \chi^{(3)} .
$$

In analogy with our notation $F$, we write

$$
F_{i}(u)=\int \check{f}_{i}(-u, v) d v .
$$

Case 1. We have

$$
\begin{aligned}
S_{j} * f_{i}(s, t) \chi^{(1)}(t) & =-j^{-4} \int r\left(s+e^{t} u\right) e^{t} F_{i}(-u) d u \chi^{(1)}(t) \\
& =-j^{-4} r_{e^{-t}} * F_{i}\left(e^{-t} s\right) \chi^{(1)}(t),
\end{aligned}
$$

the last convolution taken in $\mathbb{R}$. In Case 1 , one always has $t \in I_{j}-J_{i}$. To control the $L^{1, \infty}$ quasinorm of $\left(S_{j} * f_{i}\right) \chi^{(1)}$, we therefore estimate for $\lambda>0$ the quantity $m\left(E_{\lambda}\right)$, where

$$
E_{\lambda}=\left\{(s, t) \in G: t \in I_{j}-J_{i}, j^{-4}\left|r_{e^{-t}} * F_{i}\left(e^{-t} s\right)\right|>\lambda\right\} .
$$

Under the change of variables $x=e^{-t} s, y=e^{-t}$, the measure $m$ will correspond to $d \mu=y^{-1} d x d y$; thus

$$
m\left(E_{\lambda}\right)=\mu\left\{(x, y):-\log y \in I_{j}-J_{i}, \quad\left|r_{y} * F_{i}(x)\right|>j^{4} \lambda\right\} .
$$


But the length of the interval $I_{j}-J_{i}$ is at most $C j^{3}$, and Theorem 3 implies that

$$
m\left(E_{\lambda}\right) \leq C j^{3 / 2}\left\|F_{i}\right\|_{1} /\left(j^{4} \lambda\right) .
$$

Since $\left\|F_{i}\right\|_{1} \leq\left\|f_{i}\right\|_{1}$, this means that the $L^{1, \infty}$ quasinorm of $\left(S_{j} * f_{i}\right) \chi^{(1)}$ is at most $C j^{-5 / 2}\left\|f_{i}\right\|_{1}$. We now sum in $i$, keeping $j$ fixed. The sets $I_{j}-J_{i}, i \in \mathbb{Z}$, have $C j$-bounded overlap, in the sense that no point belongs to more than $C j$ of these sets. The same is then true for the supports of $\left(S_{j} * f_{i}\right) \chi^{(1)}, i \in \mathbb{Z}$. But for $L^{1, \infty}$ functions whose supports have $N$-bounded overlap, it is easy to see that

$$
\left\|\sum \phi_{k}\right\|_{1, \infty} \leq C N \sum\left\|\phi_{k}\right\|_{1, \infty} \text {. }
$$

(Here the factor $N$ can actually be improved to $\log N$.) This implies

$$
\left\|\sum_{i}\left|S_{j} * f_{i}\right| \chi^{(1)}\right\|_{1, \infty} \leq C j^{-3 / 2} \sum_{i}\left\|f_{i}\right\|_{1}=C j^{-3 / 2}\|f\|_{1} .
$$

To sum in $j$, we observe that the coefficients $j^{-3 / 2}$ in (12) form a sequence in $\ell \log \ell$. One can thus use the addition theorem of $L^{1, \infty}$; see Stein and N. Weiss [19, Lemma 2.3]. The result is that

$$
\left\|\sum_{j} \sum_{i}\left|S_{j} * f_{i}\right| \chi^{(1)}\right\|_{1, \infty} \leq C\|f\|_{1} .
$$

Case 2 gives nothing.

Case 3. The argument leading to (10) also implies that for each $t$

$$
\left|\left\{s:\left|S_{j} * f_{i}(s, t)\right|>\lambda\right\}\right| \leq C \lambda^{-1} j^{-4} e^{t}\left\|f_{i}\right\|_{1}, \quad \lambda>0 .
$$

Case 3 occurs precisely when $t \in \partial I_{j}-J_{i}$, and $\partial I_{j}-J_{i}$ is a set of measure $2 j^{2}$. Thus

$$
\begin{aligned}
& m\left\{(s, t):\left|S_{j} * f_{i}(s, t)\right| \chi^{(3)}>\lambda\right\} \\
& \quad \leq C \lambda^{-1} j^{-4} \int_{\partial I_{j}-J_{i}} e^{t}\left\|f_{i}\right\|_{1} e^{-t} d t \leq C j^{-2} \lambda^{-1}\left\|f_{i}\right\|_{1} .
\end{aligned}
$$

This means that

$$
\left\|\left(S_{j} * f_{i}\right) \chi^{(3)}\right\|_{1, \infty} \leq C j^{-2}\left\|f_{i}\right\|_{1} .
$$

For each fixed $j$, the sets $\partial I_{j}-J_{i}, i \in \mathbb{Z}$, and hence also the supports of $\left(S_{j} * f_{i}\right) \chi^{(3)}$, have $C$-bounded overlap. Summing as before, we get

$$
\left\|\sum_{i}\left|S_{j} * f_{i}\right| \chi^{(3)}\right\|_{1, \infty} \leq C j^{-2}\|f\|_{1}
$$

and

$$
\left\|\sum_{j} \sum_{i}\left|S_{j} * f_{i}\right| \chi^{(3)}\right\|_{1, \infty} \leq C\|f\|_{1} .
$$

Since $S * f=\sum_{j} \sum_{i} S_{j} * f_{i}$, we can combine this with (13) and obtain

$$
\|S * f\|_{1, \infty} \leq C\|f\|_{1} .
$$

This is the conclusion of Theorem 2. 
Remark. The following example shows that left convolution with the kernel $|S|$ does not define a weak type $(1,1)$ operator. We assume for convenience that 0 is in the support of $r$ considered as a distribution, but it is easy to see that the argument given below extends to any nonzero integrable $r$. Define $f \in L^{1}(G)$ by

$$
\check{f}=\chi_{[-R, R]} \otimes \chi_{[-\log R, \log R]},
$$

where $R>0$ will be large. Then

$$
|S| * f(s, t)=\int_{-R}^{R}\left|r\left(s+e^{t} u\right)\right| e^{t} d u \int_{|v|<\log R, v+t<-1} \frac{d v}{|t+v|} .
$$

If $-\log R<t<0$, the inner integral here is taken over the interval $-\log R<v<$ $-1-t$, and so its value is at least $\int_{1}^{\log R} d \rho / \rho=\log \log R$. For these $t$, we thus get

$$
|S| * f(s, t) \geq\left|r_{e^{-t}}\right| * \chi_{[-R, R]}\left(e^{-t} s\right) \log \log R,
$$

and $\left|r_{e^{-t}}\right| * \chi_{[-R, R]} \geq c$ in $(-R, R)$. Changing variables as before to $x=e^{-t} s, y=$ $e^{-t}$, we get

$$
|S| * f \geq c \log \log R
$$

for $|x| \leq R, 1<y<R$. Since $m$ corresponds to $\mu$ in the $x, y$ variables, we see that the $L^{1, \infty}(m)$ quasinorm of $|S| * f$ is at least $c R \log R \log \log R$. The $L^{1}(m)$ norm of $f$ equals the $L^{1}\left(m_{r}\right)$ norm of $\check{f}$, which is $4 R \log R$. Thus the weak type $(1,1)$ inequality is violated for large $R$.

\section{Proof of Theorem 3}

Lemma 8. The g-function

$$
g(f)(x)=\left(\int_{0}^{\infty}\left|r_{y} * f(x)\right|^{2} \frac{d y}{y}\right)^{1 / 2}
$$

defines an operator of weak type (1,1), in the sense that $\|g(f)\|_{1, \infty} \leq C\|f\|_{1}$ for $f \in L^{1}(\mathbb{R})$.

Proof. We can easily adapt the standard argument via vector-valued singular integrals, as presented e.g. in Stein [18, IV.1]. Let $\mathcal{H}$ be the Hilbert space $L^{2}\left(\mathbb{R}_{+} ; d y / y\right)$. Consider the vector-valued operator which maps a function $f \in C_{0}^{\infty}(\mathbb{R})$ to the $\mathcal{H}$ valued function in $\mathbb{R}$ whose value at $x \in \mathbb{R}$ is the element of $\mathcal{H}$ given by $y \rightarrow r_{y} * f(x)$. This can be seen as the convolution of $f$ with the $\mathcal{H}$-valued kernel $R$ defined by $(R(x))(y)=r_{y}(x), x \in \mathbb{R}, y>0$. It must be verified that $R$ is a singular integral kernel of Calderón-Zygmund type.

We start with a simple estimate for the Fourier transform $\hat{r}$. Since the integral of $r$ vanishes, one has

$$
\hat{r}(\xi)=\int\left(e^{-2 \pi i x \xi}-1\right) r(x) d x .
$$

We split this integral as $\int_{|x|<1 /|\xi|}+\int_{|x|>1 /|\xi|}$. The fact that $r(x)=O\left(|x|^{-1-\epsilon}\right)$ at infinity easily implies that for small $\xi$ these integrals are bounded by $C|\xi|^{\epsilon}$. Thus

$$
\hat{r}(\xi)=O\left(|\xi|^{\epsilon}\right) \quad \text { as } \quad \xi \rightarrow 0 .
$$

Clearly $\hat{R}$ is $\mathcal{H}$-valued and given by

$$
(\hat{R}(\xi))(y)=\hat{r}(y \xi)
$$


We get

$$
\sup _{\xi \in \mathbb{R}}\|\hat{R}(\xi)\|_{\mathcal{H}}^{2}=\sup _{\xi \in \mathbb{R}} \int|\hat{r}(y \xi)|^{2} \frac{d y}{y}=\int|\hat{r}(y)|^{2} \frac{d y}{y}<\infty,
$$

because of (14). Further,

$$
\|R(x)\|_{\mathcal{H}}^{2}=\int \frac{1}{y^{2}}\left|r\left(\frac{x}{y}\right)\right|^{2} \frac{d y}{y}=|x|^{-2} \int|w||r(w)|^{2} d w=C|x|^{-2},
$$

and similarly

$$
\left\|\frac{\partial}{\partial x} R(x)\right\|_{\mathcal{H}}^{2}=|x|^{-4} \int|w|^{3}\left|r^{\prime}(w)\right|^{2} d w=C|x|^{-4} .
$$

Taking square roots, we get the standard estimates, and the lemma follows.

Remark. The ordinary $g$-function is defined in terms of the Poisson integral and its gradient. This is similar in our case, but one must use a primitive function of $r$ instead of the Poisson kernel $\pi^{-1}\left(1+x^{2}\right)^{-1}$ of the upper half-plane. Notice that in our main case $r(x)=x\left(1+x^{2}\right)^{-3 / 2}$ this means replacing the Poisson kernel by its square root.

To prove Theorem 3 , we introduce for $f \in L^{1}(\mathbb{R})$ and $\lambda>0$

$$
J_{\lambda}(x)=\int_{\left\{y \in\left[a, e^{N} a\right]:\left|r_{y} * f(x)\right|>\lambda\right\}} \frac{d y}{y} .
$$

Then

$$
\begin{aligned}
& \mu\left\{(x, y): a \leq y \leq e^{N} a,\left|r_{y} * f(x)\right|>\lambda\right\} \\
= & \int J_{\lambda}(x) d x=\int_{0}^{N}\left|\left\{x: J_{\lambda}(x)>\alpha\right\}\right| d \alpha,
\end{aligned}
$$

the second equality because of the obvious bound $J_{\lambda}(x) \leq N$ for all $\lambda$ and $x$. By the definition of $g(f)$,

$$
g(f)(x)^{2} \geq \lambda^{2} J_{\lambda}(x)
$$

and Lemma 8 implies

$$
\begin{aligned}
\int_{0}^{N} \mid\left\{x: J_{\lambda}(x)\right. & >\alpha\}\left|d \alpha \leq \int_{0}^{N}\right|\{x: g(f)(x)>\lambda \sqrt{\alpha}\} \mid d \alpha \\
& \leq \int_{0}^{N} \frac{C}{\lambda \sqrt{\alpha}}\|f\|_{1} d \alpha \leq C \sqrt{N} \frac{1}{\lambda}\|f\|_{1} .
\end{aligned}
$$

From this and (15), Theorem 3 follows.

\section{REFERENCES}

[1] G. Alexopoulos, An application of homogenization theory to harmonic analysis: Harnack inequalities and Riesz transforms on Lie groups of polynomial growth. Can. J. Math. 44 (1992), 691-727. MR 93j:22006

[2] J.-Ph. Anker, Sharp estimates for some functions of the Laplacian on noncompact symmetric spaces, Duke Math. J. 65 (1992), 257-297. MR 93b:43007

[3] D. Bakry, Etude des transformations de Riesz dans les variétés riemanniennes à courbure de Ricci minorée. In Séminaire de Probabilité XXI, J. Azéma et al. (eds), Lecture Notes in Mathematics 1247, Springer-Verlag 1987, 137-172. MR 89h:58208

[4] M.S. Birman and M.Z. Solomjak, Spectral theory of self-adjoint operators in Hilbert space. (Mathematics and its applications (Soviet series) Reidel, Dordrecht 1987. MR 93g:47001 
[5] R. Burns, A.F.M. ter Elst and D.W. Robinson, $L_{p}$-regularity of subelliptic operators on Lie groups. J. Operator Theory 31 (1994), 165-187. MR 96a:22013

[6] Th. Coulhon and X.Th. Duong, Riesz transforms for $1 \leq p \leq 2$. To appear in Trans. Amer. Math. Soc. CMP 97:15

[7] R.E. Edwards and G.I. Gaudry, Littlewood-Paley and multiplier theory. Ergebnisse der Mathematik und ihrer Grenzgebiete 90, Springer-Verlag, Berlin Heidelberg New York 1977. MR $\mathbf{5 8 : 2 9 7 6 0}$

[8] G.I. Gaudry, T. Qian and P. Sjögren, Singular integrals related to the Laplacian on the affine group $a x+b$. Ark. Mat. 30 (1992), 259-281.

[9] G. Gaudry and P. Sjögren, Singular integrals on Iwasawa NA groups of rank 1. J. Reine Angew. Math. 479 (1996), 39-66. MR 97k:22012

[10] Singular integrals on the complex affine group. Coll. Math. 75 (1998), 133-148. CMP 98:06

[11] Haar-like expansions and boundedness of a Riesz operator on a solvable group. To appear in Math. Zeitschr.

[12] A. Hulanicki, On the spectrum of the Laplacian on the affine group of the real line. Studia Math. 54 (1976), 199-204. MR 53:3195

[13] N. Lohoué, Comparaison des champs de vecteurs et des puissances du laplacien sur une variétés riemanniennes à courbure non positive. J. Funct. Anal. 61 (1985), 164-201. MR 86k:58117

[14] - Transformées de Riesz et fonctions de Littlewood-Paley sur les groupes non moyennables. C. R. Acad. Sci. Paris Sér. I Math. 306 (1988), 327-330. MR 89b:43008

[15] N. Lohoué and N.Th. Varopoulos, Remarques sur les transformées de Riesz sur les groupes de Lie nilpotents. C. R. Acad. Sci. Sér. I Math. 301 (1985), 559-560. MR 87b:43008

[16] W. Magnus and F. Oberhettinger, Formulas and theorems for the special functions of mathematical physics. Chelsea Publishing Company, New York 1949. MR 10:532b

[17] L. Saloff-Coste, Analyse sur les groupes de Lie à croissance polynômiale. Ark. Mat. 28 (1990), 315-331. MR 92d:22014

[18] E.M. Stein, Singular integrals and differentiability properties of functions. Princeton University Press, Princeton 1970. MR 44:7280

[19] E.M. Stein and N.J. Weiss, On the convergence of Poisson integrals. Trans. Amer. Math. Soc. 140 (1969), 35-54. MR 39:3024

[20] J.-O. Strömberg, Weak type $L^{1}$ estimates for maximal functions on non-compact symmetric spaces and biinvariant convolutions. Unpublished manuscript, 1979.

[21] W Weak type $L^{1}$ estimates for maximal functions on non-compact symmetric spaces. Ann. Math. 114 (1981), 115-126. MR 82k:43010

Department of Mathematics, Chalmers University of Technology and Göteborg UniVERSity, S-412 96 Göteborg, Sweden

E-mail address: peters@math.chalmers.se 